Hydroécol. Appl. (1992) Tome 4 Vol 2, pp. 1-7

\title{
Résistance bactérienne aux métaux lourds chez les souches isolées des moules du littoral Atlantique Marocain
}

\section{Bacterial resistance to heavy metals in isolated strains from the mussels of the Atlantic Moroccan coast}

\author{
Attrassi Benaissa* et Saghi Mohamed ${ }^{\star \star}$ \\ * Université Mohamed $I^{\theta r}$, Faculté des Sciences, Département de Biologie, Laboratoire de \\ Microbiologie, Oujda, Maroc \\ "* Université sidi Mohamed Ben Abdellah, Faculté des Sciences, Département de Biologie, La- \\ boratoire de Biotechnologie, Fès, Maroc
}

Résumé. - La résistance bactérienne aux métaux lourds a été examinée sur trois sites de la côte atlantique marocaine entre Kenitra et Temara. Les souches résistantes au $\mathrm{Hg}^{++}$et au $\mathrm{Cd}^{++}$ont été isolées uniquement de moules provenant des sites pollués par les égoûts ou les abattoirs municipaux de Rabat; deux souches hautement résistantes ont été identifiées: S. paratyphi B el Aeromonas M1/11, leurs CMl atteignent 400 ppm $\mathrm{CdCl}_{2}$ et $100 \mathrm{ppm} \mathrm{HgCl}$ respectivement et leurs taux de croissance ne sont affectés qu'à des concentrations supérieures à $10 \mathrm{ppm} \mathrm{HgCl}_{2}$ ou $50 \mathrm{ppm} \mathrm{CdCl}_{2}$. Ces concentrations sont bactéricides pour toutes les souches isolées de l'eau de mer. Les moules constituent un microenvironnement favorable à l'apparition des bactéries multirésistantes aux antibiotiques et métaux lourds.

La résistance d'Aeromonas M1/11 au cadmium et au mercure est portée par un seul plasmide de $20 \mathrm{~kb}$, qui lorsqu'il est transféré à $E$. coli exprime une mercure-réductase NADPH dépendante.

Mots-clés. - Pollution marine - Résistance bactérienne aux métaux lourds - Plasmide - Mercure réductase.

Abstract. - Three marine sites between Kenitra and Temara have been looked for heavy metals resistant bacteria. Strains which are resistant to $\mathrm{Hg}^{++}$and $\mathrm{Cd}^{++}$have been isolated only from mussels; two highly resistant strains have been identified: $S$. paratyphi $B$ and 
Aeromonas $\mathrm{M} 1 / 11$, their $\mathrm{CMI}$ reaches $400 \mathrm{ppm} \mathrm{CdCl}_{2}$ and $100 \mathrm{ppm} \mathrm{HgCl}_{2}$ respectively and their growth rates are only affected at concentrations higher than $10 \mathrm{ppm} \mathrm{HgCl}_{2}$ ou $50 \mathrm{ppm} \mathrm{CdCl}_{2}$. Those concentrations killed all bacterial strains isolated from water and marine sediments. Mussels constitute a favorable microsurrounding for the appearance of multiresistant bacteria to antibiotics and heavy metals.

Cadmium and mercury resistant Aeromonas M1/11 are genetically linked and carried by one plasmid of $20 \mathrm{~kb}$, which expresses a mercure-reductase NADPH dependant when transfered to $E$. coli.

Key words. - Marine pollution - Heavy metals resistant bacteria - Plasmid - Mercure reductase.

\section{INTRODUCTION}

Les milieux aquatiques sont aujourd'hui le réceptable d'effluents et de déchets industriels et urbains souvent toxiques pour les organismes vivants. Les métaux lourds tels que le cadmium et le mercure peuvent atteindre l'homme par la voie des chaines alimentaires, soit par l'intermédiaire des plantes quand les boues organiques des stations d'épuration riches en métaux lourds sont utilisées comme fertilisants (Kurek et al., 1982), soit par des organismes bioaccumulateurs consommés tels que les moules Mahyaoui et Saghi, 1987).

Ces métaux toxiques sont connus comme de puissants inhibiteurs de plusieurs activités enzymatiques (Williams, 1976). Les microorganismes ont cependant une grande capacité d'adaptation à des environnements hostiles par l'apparition de souches résistantes. Ainsi le mercure $\mathrm{Hg}^{++}$, toxique, peut être réduit en $\mathrm{Hg}(\mathrm{O})$, forme volatile non toxique; cette fonction est codée par des gènes portés par des plasmides de haut poids mo- léculaire (Barkay, 1987; Bale et al., 1988). Notre étude s'inscrit dans les recherches sur la résistance aux métaux. Nous avons étudié la résistance de souches bactériennes marines à des métaux lourds et la détoxification en relation avec l'accumulation de mercure et cadmium dans des moules prélevées dans des sites contaminés par des rejets d'égoûts et d'abattoirs municipaux.

\section{MATÉRIELS ET MÉTHODES}

\section{Prélèvement des échantillons}

Les échantillons d'eau, de moules et de sédiments sont aseptiquement prélevé en trois sites localisés sur la figure 1:

- Site 1: pollué par les rejets des abattoirs municipaux de Rabat.

- Site 2: pollué par les eaux usées de Rabat.

- Site 3: situé en amont de l'estuaire de l'oued Bouregreg est considéré comme "témoin". 


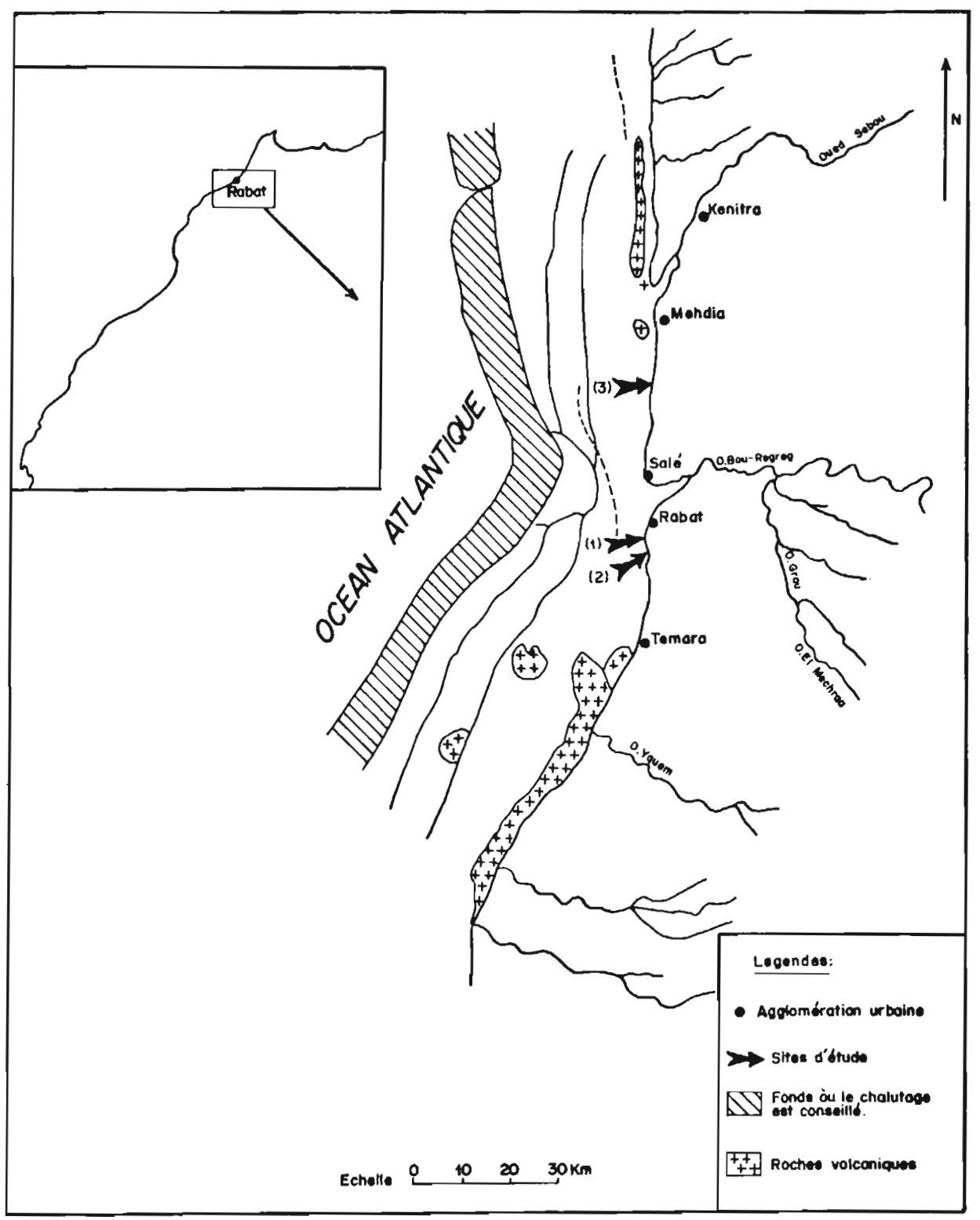

Fig. 1. - Localisation des sites d'échantillonnage.

Fig. 1. - Location of sampling sites. 


\section{Détermination des taux de croissance et des CMI}

Des solutions à différentes concentrations du métal étudié, préalablement stérilisées par filtration, sont ajoutées à une série de tubes contenant $10 \mathrm{ml}$ de milieu Y.T qu'on inocule par $10^{6}$ CFU. Après incubation à $35^{\circ} \mathrm{C}$ sous agitation de $100 \mathrm{rpm}$, les absorbances à $600 \mathrm{mn}$ sont notées toutes les demies heures. Les concentrations minimales inhibitrices sont notées après une nuit de culture.

\section{Extraction des plasmides}

Le contenu plasmidique des souches identifiées est analysé par la méthode de Maniatis et al. (1982).

\section{Transfert des marqueurs de résistance aux métaux lourds}

- En milieu liquide la conjugaison est effectuée classiquement selon Miller (1972).

- En milieu solide: la méthode décrite par Bradley et al. (1980) est utilisée.

\section{Dosage de la mercure réductase}

Un volume de $25 \mathrm{ml}$ du milieu de culture YT est inoculé avec $250 \mu \mathrm{l}$ d'une préculture d'une nuit. Lorsque la croissance arrive en phase exponentielle, l'enzyme mercure réductase est induite par l'addition de $5 \mu \mathrm{M}$ de $\mathrm{HgCl}_{2}$. La culture est poursuivie pendant 75 minutes, puis les cellules sont collectées, lavées deux fois par le tampon phosphate à $\mathrm{pH} 6.8$, $50 \mathrm{mM}$ et resuspendues dans le même tampon à une densité optique de 10 unités. Les cellules sont ensuite soniquées $3 \mathrm{mn}$ à $0^{\circ} \mathrm{C}$ avec $80 \%$ du cycle actif et une puissance de 100 watts (Sonicateur vibro-cell).

Après centrifugation à $5000 \mathrm{~g}$ pendant 30 minutes à $4{ }^{\circ} \mathrm{C}$, l'activité enzymatique du surnageant est dosée à $340 \mathrm{~nm}$ par oxydation de NADPH qui accompagne la réduction du mercure. Le mélange réactionnel comprend: 0,5 mM EDTA; 0,2 mM acétate $\mathrm{Mg}$; $0,03 \mathrm{mg} / \mathrm{ml}$ BSA et $1 \mathrm{mM}$ Lcys.

\section{RÉSULTATS ET DISCUSSION}

Les effets des métaux lourds sur 75 souches isolées des eaux, des sédiments marins et des moules, nous ont montré que la majorité des souches sont sensibles, alors que les quelques souches résistantes au $\mathrm{Cd}^{++}$ou au $\mathrm{Hg}^{++}$proviennent des moules. Ainsi, le taux de croissance d'E. coli S2/9, isolée des sédiments, décroît de façon exponentielle en fonction de la concentration de mercure et s'annule à 2 ppm.

Dans le cas du cadmium, le même phénomène est obtenu; cependant l'inhibition de la croissance n'apparaît qu'à partir de 20 ppm (figs. 2 et 3).

En revanche, la croissance d'Aeromonas $\mathrm{M} 1 / 11$, souche isolée des moules du site pollué par les abattoirs, n'est réellement inhibée qu'à 


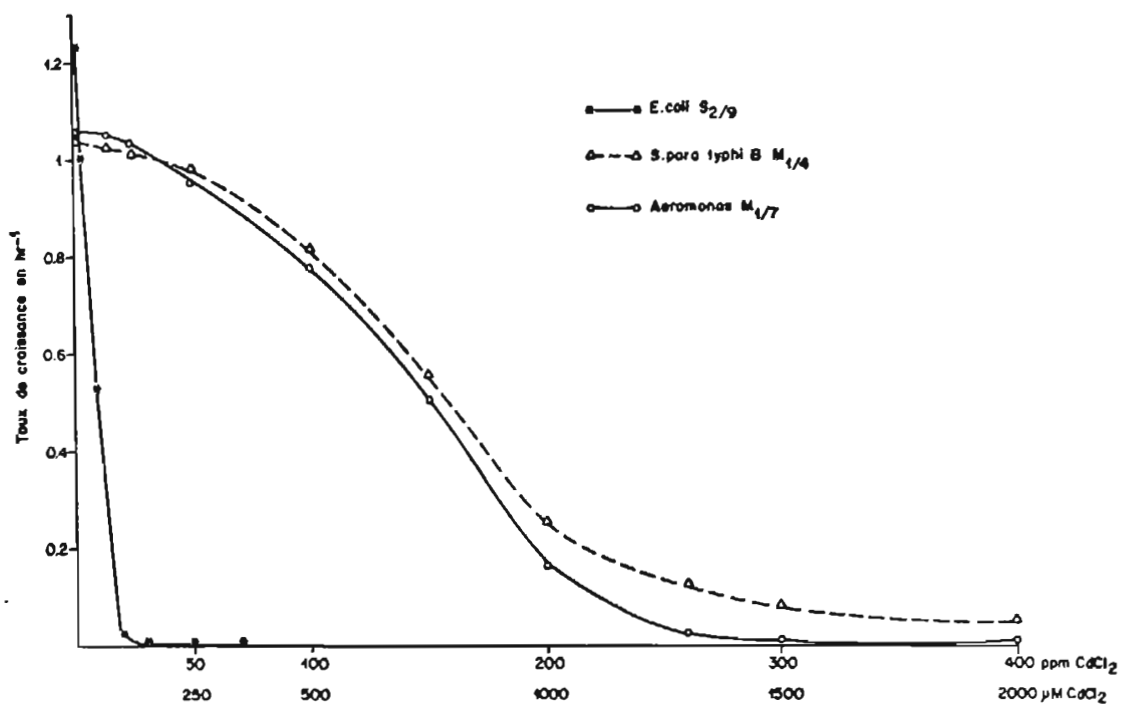

Fig. 2. - Effet de la concentration en chlorure de cadmium sur les taux de croissance d'Aeromonas $M 1 / 7, S$. paratyphy B M1/4 et $E$. coli S2/9. Le milieu Y.T contenant différentes concentrations du métal étudié est inoculé avec $10^{6} \mathrm{CFU}$ et incubé à $35^{\circ} \mathrm{C}$ sous agitation horizontale. Les taux de croissance sont déterminés graphiquement.

Fig. 2. - Effect of cadmium chloride concentration on the growth rate of Aeromonas M1/7, S. paratyphy $\mathrm{B} M 1 / 4$ and $E$. coli S2/9. Y.T medium supplemented with different concentrations of heavy metals, inoculed with $10^{6} \mathrm{CFU}$, was incubated at $35^{\circ} \mathrm{C}$ under horizontal agitation. Growth rates are graphically determined.

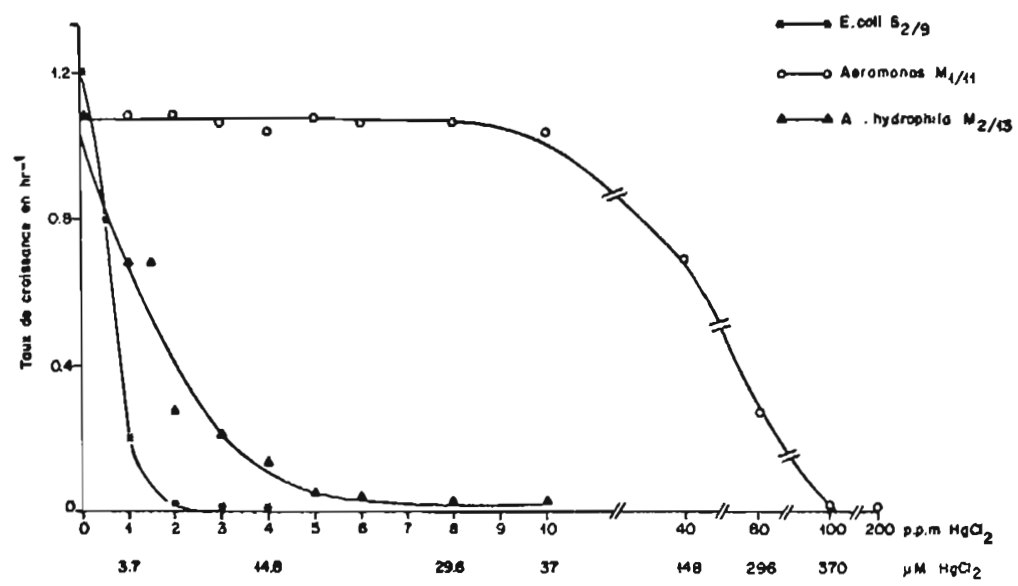

Fig. 3. - Effet de la concentration en chlorure mercurique sur les taux de croissance d'Aeromonas M1/11, Aeromonas hydrophila M2/13 et E. coli S2/9. Le milieu Y.T contenant differentes concentrations du métal étudié est inoculé avec $10^{6} \mathrm{CFU}$ et incubé à $35^{\circ} \mathrm{C}$ sous agitation horizontale. Les taux de croissance sont déterminés graphiquement.

Fig. 3. - Effect of mercury chloride concentration on growth rates of Aeromonas M1/11, Aeromonas hydrophila $\mathrm{M} 2 / 13$ and $E$. coli S2/9. Y.T medium supplemented with different concentrations of heavy metal, inoculed with $10^{6} \mathrm{CFU}$ was incubated at $35^{\circ} \mathrm{C}$ under horizontal agitation. Growth rates are graphically determined. 
des concentrations supérieures à $100 \mathrm{ppm}$ de $\mathrm{HgCl}_{2}$ ou $150 \mathrm{ppm}$ de $\mathrm{CdCl}_{2}$. Des souches monorésistantes, peuvent supporter des concentrations plus élevées en cadmium; c'est le cas d'Aeromonas M1/7 et $S$. paratyphi B M1/4 qui continuent de se multiplier à des concentrations supérieures à 250 ppm.

Dans le but d'expliquer les niveaux très élevés de résistance aux métaux lourds des souches isolées, nous avons analysé leur contenu plasmidique et nous avons observé que toutes les souches résistantes hébergent des plasmides de PM de l'ordre de $20 \mathrm{~kb}$. Les expériences de conjugaison ont montré qu'il s'agit de plasmides conjugatifs capables d'être transmis d'une espèce à une autre. Toutefois ces plasmides ne sont pas tous transmissibles en milieu liquide; c'est ainsi qu'Aeromonas M1/11 ne conjugue qu'en milieu solide. La fréquence de conjugaison dans ce cas est très faible car $E$. coli et
Aeromonas sont deux espèces génétiquement éloignées: $f=2.10^{-7}$ (tableau I).

L'isolement des souches résistantes au $\mathrm{Cd}^{++}$et $\mathrm{Hg}^{++}$à partir des moules suggère que ces organismes offrent le support nécessaire à la conjugaison et concentrent suffisamment de souches conjugatives ce qui constitue un réservoir plasmidique pouvant alimenter de manière continue des souches de coliformes. Les souches transconjugantes retrouvent le même niveau de résistance que le donneur (fig. 4).

Des analyses biochimiques nous ont montré que ces souches produisent une mercure réductase NADPH dépendante. A des concentrations subtoxiques en chlorure de mercure $(15 \mu \mathrm{M})$, l'extrait brut du donneur et des transconjugants réduisent ce métal à un taux voisin de $1,4 \mu$ mole/mg de protéine (fig. 5).

Tableau I. - Conjugaison interspécifique entre différentes espèces isolées du littoral marocain.

$\mathrm{D}$ : Donatrice et R: Réceptrice.

$\mathrm{CdCl}_{2}: 100 \mu \mathrm{g} / \mathrm{ml} ; \mathrm{HgCl}_{2}: 20 \mu \mathrm{g} / \mathrm{ml}$

Streptomycine (str): $100 \mu \mathrm{g} / \mathrm{ml}$ et Tetracycline (Tet): $25 \mu \mathrm{g} / \mathrm{ml}$.

Tableau I. - Interspecific conjugation between different species isolated from moroccan seaboard.

D: Donor et R: Recipient.

$\mathrm{CdCl}_{2}: 100 \mu \mathrm{g} / \mathrm{ml} ; \mathrm{HgCl}_{2}: 20 \mu \mathrm{g} / \mathrm{ml}$

Streptomycine (str) : $100 \mu \mathrm{g} / \mathrm{ml}$ and Tetracycline (Tet) : $25 \mu \mathrm{g} / \mathrm{ml}$.

\section{Croisements \\ $\mathrm{D}, \mathrm{R}$}

S. paratyphi B $\times$ E coli S2/9

Aeromonas M1/10, E. coli S2/9

Aeromonas M1/11 a E. coli S2/9

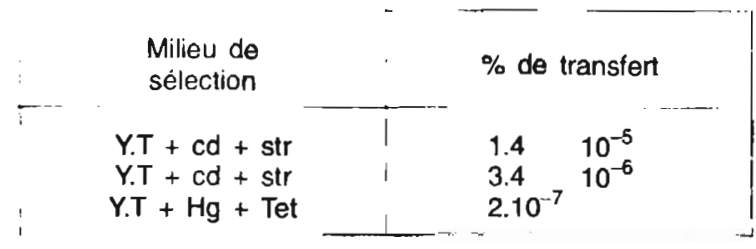




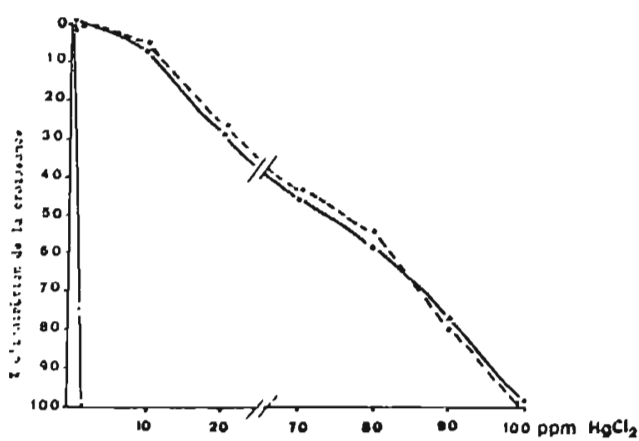

Fig. 4. - Inhibition de la croissance d'E. coli S2/9 (transconjugante) $\boldsymbol{\Delta}--\boldsymbol{\Delta}$, Aeromonas M1/11 (donatrice) - - et E. coli S2/9 o o (réceptrice) par $\mathrm{HgCl}_{2}$ dans le milieu Y.T au bout de $12 \mathrm{~h}$ à $37^{\circ} \mathrm{C}$ et sous agitation de $100 \mathrm{rpm}$. Fig. 4. - Growth inhibition of E. coli $\$ 2 / 9$ (transconjugante) $\mathbf{\Delta}--\boldsymbol{\Delta}$. Aeromonas M1/11 (Donor) - - and E. coli S2/9 o-o (Recipient) by $\mathrm{HgCl}_{2}$ in Y.T medium after $12 \mathrm{~h}$ at $37^{\circ} \mathrm{C}$ under agitation of $100 \mathrm{rpm}$.

La haute capacité de résistance bactérienne au mercure et au cadmium montre le rôle fondamental que peuvent jouer ces plasmides dans la réponse rapide des populations naturelles aux stress.

\section{RÉFÉRENCES \\ BIBLIOGRAPHIQUES}

Bale MM.J., Fry J.C. and Day M.J., 1988. Transfer and occurence of large mercury resistance plasmids in river epilithon. Appl. and Env. Microbiol., 54 (4), 972-978.

Barkay T., 1987. Adaptation of aquatic Microbiol communities to $\mathrm{Hg}^{++}$stress. Appl. and Env. Microbiol., 53 (12), 2725-2732.

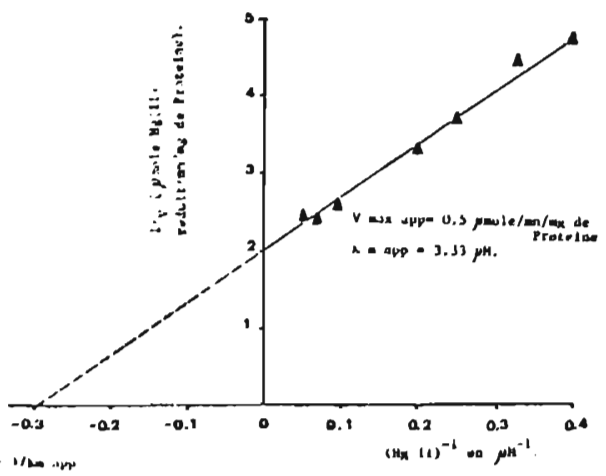

Fig. 5. - Représentation Burk de la réduction du mercure $\mathrm{Hg}(\mathrm{II})$ à partir d'extrait d'Aeromonas $\mathrm{M} 1 / 11$ et en présence de $0.2 \mathrm{mM}$ NADP réduit. Fig. 5. - Lineweaver Burk representation of mercury (HGII) reduction from Aeromonas M1/11 extract at $0.2 \mathrm{mM} \mathrm{NADPH} 2$.

Bradley D.E., Taylor D.E. and Cohen D.R., 1980. J. Bacteriol, 143, 1466-1470.

Kurek E., Czaban J. and Ballag J.M., 1982. Sorption of cadmium bay microorganismes in competition with other soil constituents. Appl. and Env. Microbiol., 43 (5), 1011-1015.

Mahyaoui M. et Saghi M., 1987. Étude de la pollution de l'eau de mer par les métaux lourds. Rev. Int. Océanogr. Med. Tomes LXXXVII-LXXXVIII.

Maniatis T., Fritach E.F. and Sambrook J., 1982. Molecular cloning: a laboratory manual. Cold. spring. Harbor Laboratory. Cold. Spring. Harbor., New York, $504 \mathrm{pp}$.

Miller J.H., 1972. Experiments in molecular genetics. Cold. spring Harbor Laboratory. New York, 436 pp.

Williams R.J.P., 1967. Les métaux lourds dans les systèmes biologiques. Endeavour, vol. XXVI, $n^{\circ}$ 98, 96-101. 